\title{
A Study on the Design and Implementation of Facial Recognition Application System
}

\author{
Yoon Young Park ${ }^{1}$, YongKeum $\mathrm{Choi}^{2}$ and KyungOh Lee ${ }^{1 *}$ \\ ${ }^{1}$ Department of Computer Engineering, Sunmoon University, Korea \\ ${ }^{2}$ Department of Dental hygiene, Sunmoon University, Korea \\ *Corresponding Author:leeko@sunmoon.ac.kr \\ \{yypark, jennychoi, leeko\}@sunmoon.ac.kr
}

\begin{abstract}
Face recognition systems gain flexibility and cost efficiency while being integrated into a wireless network. In this paper, we describe a study on the implementation of facial recognition application system using wireless devices. The facial recognition system transmits the pictures photographed by wireless terminals or smart phones and recognizes/authenticates the faces. This system can be applied to several areas such as indolence management, access management and attendance management. The previous facial recognition systems are mostly based on the wire system but our system uses mobile terminals or smart phones.
\end{abstract}

Keywords: facial recognition, mobile terminal, smart phone, detection

\section{Introduction}

Face detection is a computer technology that determines the locations and sizes of human faces in arbitrary (digital) images. It detects facial features and ignores anything else, such as buildings, trees and bodies [1].

Some facial recognition algorithms identify facial features by extracting landmarks, or features, from an image of the subject's face. For example, an algorithm may analyze the relative position, size, and/or shape of the eyes, nose, cheekbones, and jaw. These features are then used to search for other images with matching features. Other algorithms normalize a gallery of face images and then compress the face data, only saving the data in the image that is useful for face detection. A probe image is then compared with the face data. One of the earliest successful systems is based on template matching techniques applied to a set of salient facial features, providing a sort of compressed face representation [2].

Recognition algorithms can be divided into two main approaches, geometric, which looks at distinguishing features, or photometric, which is a statistical approach that distills an image into values and compares the values with templates to eliminate variances. Popular recognition algorithms include Principal Component Analysis using eigenfaces, Linear Discriminate Analysis, Elastic Bunch Graph Matching using the Fisherface algorithm, the Hidden Markov model, the Multilinear Subspace Learning using tensor representation, and the neuronal motivated dynamic link matching.

Face recognition includes face verification and face identification. Face verification is oneto-one matching, which has been implemented in mobile phone and personal computer login systems, by OMRON, Oki Electric, and FaceCode, etc. Face identification is one-to-many matching, where a huge database needs to be compared, and thus more challenging. It's 
envisioned in the future that face identification can be also carried out with a mobile terminal interface, which captures face images and accesses face recognition processor or database through wireless network. For instance, after a policeman captures a suspect's facial image, $\mathrm{s} / \mathrm{he}$ can access the face recognition system remotely through a hand-held unit and identify the suspect on the spot. It will be more interesting if an un-manned surveillance system works independently and sends out alerts whenever somebody suspicious is in vision. The challenges in realizing this vision include: A wireless face recognition system requires considerable energy, computation, and bandwidth for image acquisition, processing, and transmission. Face recognition techniques need to be robust and accurate, and it should scale with the size of user group [3, 4].

Face detection plays an important role in applications such as human computer interface, face recognition video surveillance and face image database management. With the pervasive presence of low-cost, high quality cameras on mobile devices, we are witnessing the beginning of an explosive growth of embedded computer vision applications, such as mobile augmented reality, mobile image search and real-time image content analysis [5].

There is some relevant work on wireless face recognition: Zaeri, Mokhtarian and Cherri discuss face recognition for wireless surveillance systems [6]. Rajani and Yan propose a prioritized transmission scheme for a wireless system especially designed for face recognition [7]. Ikdong, Jaechang, Jason and Wayne implement a wireless face recognition system based on ZigBee transmission protocol and Eigenface method with low power consumption [8]. Though not designing for a face recognition system, Robert and Leonard propose a simulation framework and performance criteria for a distributed sensor processing over an ad hoc wireless network [9], where a model of three conceptual layers in a wireless sensor network is proposed. Based on this model, this paper proposes a distributed wireless network prototype to carry out face identification task by optimally allocating the resources within the network. The face recognition technique used in this paper is subspace-based modular processing with score and decision level fusion, where the features are selected by statistical step-wise simulation, and the fusion results are shown to be superior to using either the whole face or modules alone [10].

We develop a mobile recognition system with mobile devices and smartphones as Figure 1.

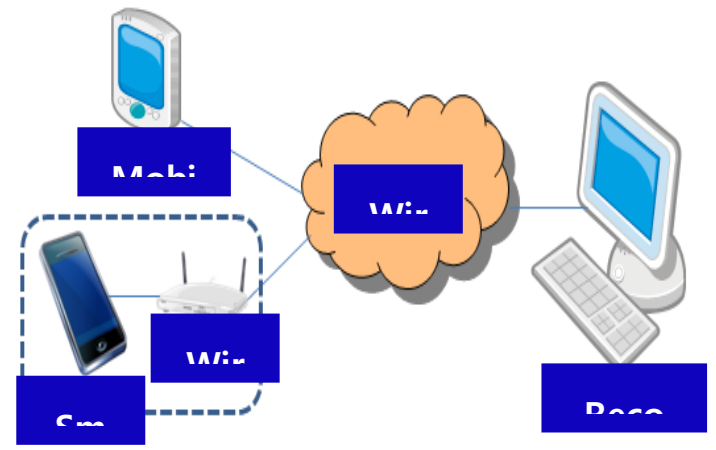

Figure 1. General System Architecture

The motivation of this research is that there are many benefits from constructing a wireless face recognition system. A wireless network is very suitable for constructing a flexible face recognition system, because a wireless setup incurs minimal modification to existing infrastructures, and a wireless network provides scalable and flexible monitoring region. If a 
wireless face recognition system has been set up, once the security level changes and the network needs to be moved, the wireless network can be moved and reused easily. Face recognition enhances the functionality and security of the wireless network, whilst a face recognition system is integrated with existing systems. Face recognition provides identity and expression information for event analysis to constitute a smart environment, and face recognition can replace or concert with other security measures to enhance the security.

The rest of the paper is organized as follows. Chapter 2 reviews the face recognition systems. Chapter 3 discusses the process of facial recognition. Chapter 4 provides implementation details, and Chapter 5 concludes this paper.

\section{Related Works}

Without considering the wireless network, the diagram of the core face recognition system is shown in Figure 2. In enrollment, the images of the registered users are processed into templates of caricatures by the specific algorithms of the face recognition system, and these templates are stored. The templates can be regarded as the transformed user images encoded by the corresponding processing techniques. The processing techniques and the templates are adjusted concurrently. In verification or identification, the face recognition system receives a new image, defines and stores the new image by the same algorithm, and compares to the templates. The decision process may incorporate all kinds of classifiers. If the classifier is a learning algorithm and its structure needs to be trained such as the neural network or Bayesian network, the enrollment database may be split into two parts, one for constructing the templates, and one for learning the classifier structure [1].

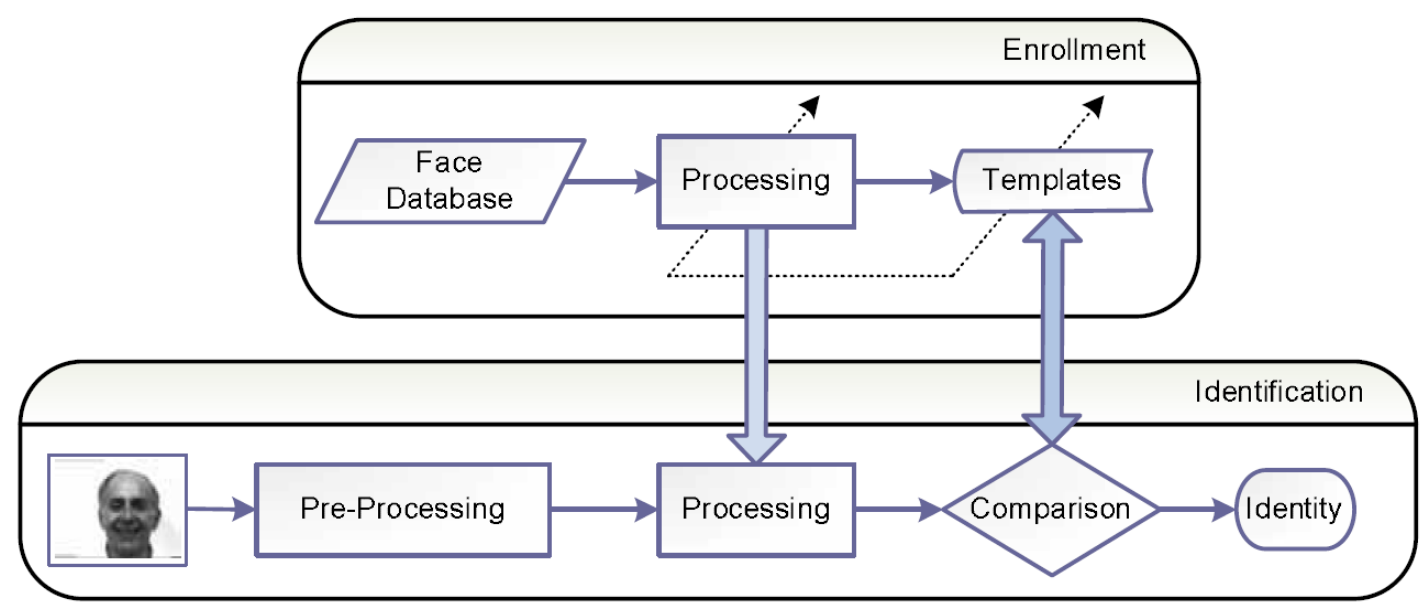

Figure 2. General Diagram of the Face Recognition System

Face recognition techniques can be roughly classified into the following categories. Interested readers are encouraged to get more references from reading these provided references.

- Template based: A typical template-based method is PCA (Principle Component Analysis) based eigenface method, which uses holistic information of the face. The eigenfaces are extracted from the training images [11]. The face images of the people on the watch list are projected onto the eigenface space, and the coordinates are stored as templates to compare with testing images. There are other transforms based on LDA (Linear Discriminant 
Analysis) or ICA (Independent Component Analysis), and the templates are associated with the transforms.

- Feature based: Features are descriptions or quantitative measurements of local facial features such as eyes, nose and mouth for direct comparisons. The local features need not to be organs, but they are meaningful objects occupying partial image. The local features segmented from the facial image do not have to be used altogether; the features can be further selected for better performance. The features can be generalized as responses to Gabor filters etc.

- Rule based: A learning algorithm, such as support vector machine (SVM), decision tree, neural network or Bayesian network, is trained on the available dataset, which constitutes an explicit or implicit set of rules. The rules are evaluated on the testing image to reach a final decision.

- Model based: The most popular models include elastic-bunch-graph (EBG) model and hidden Markov model. In EBG analysis, the bunch graph is constructed from a small set of sample image graphs. Recognition is based on a straightforward comparison of image graphs. In hidden Markov model analysis, the strips tessellating the facial image are assumed to be related by the hidden Markov model. In both models, the model parameters are fitted to the training images, and each subject has a distinctive model. The recognition is based on the fitting of the models. Generally in 3D face modeling and analysis, the model fitting is also essential.

- Module based: Facial modules are similarly defined or detected as local features, but modules are analyzed as self-contained components. The features, scores or decisions from modules can be also combined together to reach a fused result. The fusion at both score level and decision level is shown to improve the recognition performance.

\section{Process of Facial Recognition}

Facial recognition is the automatic processing of digital images which contain the faces of individuals for the purpose of identification, authentication/verification or categorisation 1 of those individuals. The process of facial recognition itself is comprised of a number of discrete sub-processes [2]:

a) Image acquisition: The process of capturing the face of an individual and converting to a digital form (the digital image). In an online and mobile service the image may have been acquired in a different system, e.g., taking a photograph with a digital camera which is then transferred to an online service.

b) Face detection: The process of detecting for the presence of a face within a digital image and marking the area.

c) Normalization: The process to smooth variations across detected facial regions, e.g., converting to a standard size, rotating or aligning color distributions.

d) Feature extraction: The processing of isolating and outputting repeatable and distinctive readings from the digital image of an individual. Feature extraction can be holistic2, feature-based or a combination of the two methods. The set of key features may be stored for later comparison in a reference template.

e) Enrolment: If this is the first time an individual has encountered the facial recognition system the image and/or reference template may be stored as a record for later comparison. 
f) Comparison: The process of measuring the similarity between a set of features (the sample) with one previously enrolled in the system. The main purposes of comparison are identification and authentication/verification. A third purpose of comparison is categorization which is the process of extracting features from an image of an individual in order to classify that individual in one or several broad categories (e.g., age, gender, color of clothes, etc.).

\section{Implementation Details}

Our facial recognition application system can enroll face pictures into the PC server and recognize faces with mobile terminals or smartphones as shown in Figure 1.

\subsection{Implementation of Facial Recognition Application}

At first we implement mobile embedded wireless terminal for facial recognition. We design the exterior of the embedded wireless terminal, build the hardware board and implement a prototype with GUI, communication modules and Camera facilities. The implemented embedded wireless terminal is connected to the server as in Figure 1 and we test it with the attendance check system through facial recognition. Our wireless facial recognition system is easy to install, light-weighted and small sized. In our research we design the system to produce hardware with a reasonable cost and it is possible to construct the minimum cost facial recognition system.

Next step is recognition of faces and authentication using mobile terminals or smartphones. Mobile terminals or smartphones can be connected to server by a wireless AP and pictures taken by smartphones can be sent to server through wireless network. Smartphone users can download the facial recognition application without any cost and can apply it to many areas easily. The application sends photos taken by smartphone to server and recognizes location of user. If it is not the registered location, the server denies the authentication. So the users should send the picture on the registered spots.

\subsection{Implementation of Facial Recognition Kiosk and mobile terminal}

We design and implement a kiosk to run the facial recognition application system. The kiosk is the main part of facial recognition system and has several functions as follows.

- Touch screen GUI

- Registration/authentication of face pictures on the server

- Registration/authentication of face pictures on the mobile terminal and smartphone

- Management function to maintain the facial application system

The mobile terminal implemented has the characteristics as follows.

- Registration of face pictures taken by the mobile terminal on the server

- Low cost implementation of mobile terminal

- Ease of use with only minimal essential modules

- Light-weight small size terminal 


\subsection{Basic modules of facial recognition server}

Our facial recognition application system can enroll face pictures into the PC server and recognize with mobile terminals and smartphones as shown in Figure 3. Main modules of smartphones and wireless terminals are Camera Module and Communication Module.

- Camera Module - This module takes picture of face and saves the files with JPG format.

- Communication Module - This module communicates with the PC server to send pictures and get the recognition result.

Main modules of the Server are Communication Module, Authentication Module, Registration Module and Storage Module.

- Authentication Module - This module recognizes face with the transmitted pictures using the facial recognition engine.

- Registration Module - This module register the face of users and facial recognition patterns.

- Storage Module - This module saves the user information and status into the database.

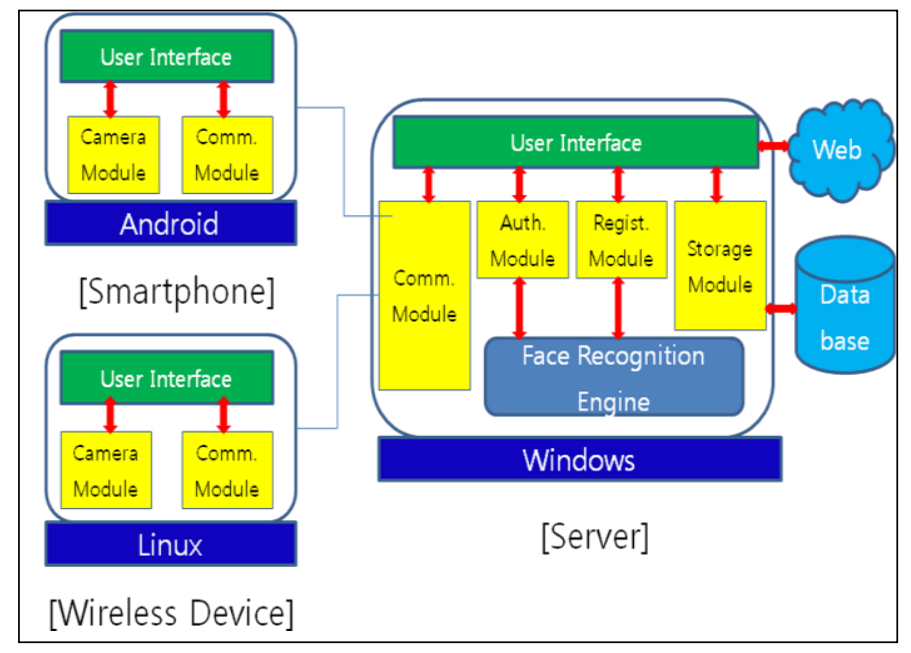

Figure 3. System Architecture

\subsection{Sequential Diagram of Facial Recognition System}

Face registration can be done by $\mathrm{PC}$, mobile terminal or smartphone. We describe the sequential diagram of face registration in Figure 4. User can register face pictures using server, mobile terminal or smartphone. The sequential diagram of face authentication is given in Figure 5. User can authenticate using server, mobile terminal or smartphone. 


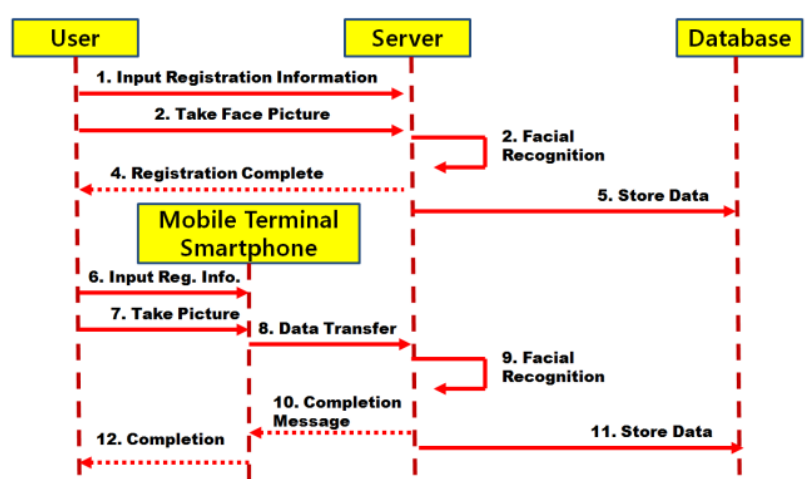

Figure 4. Sequential Diagram of Registration

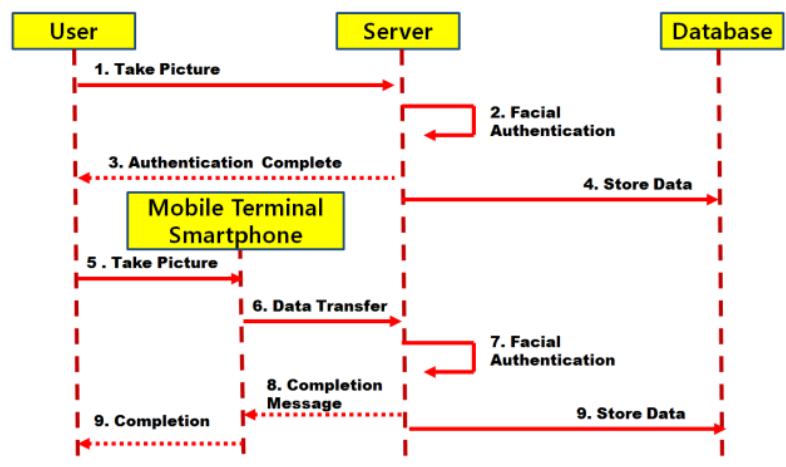

Figure 5. Sequential Diagram of Authentication

\subsection{Sample Screen Shot of the System}

Figure 6 and 7 show examples of face authentication using server and mobile terminal respectively.

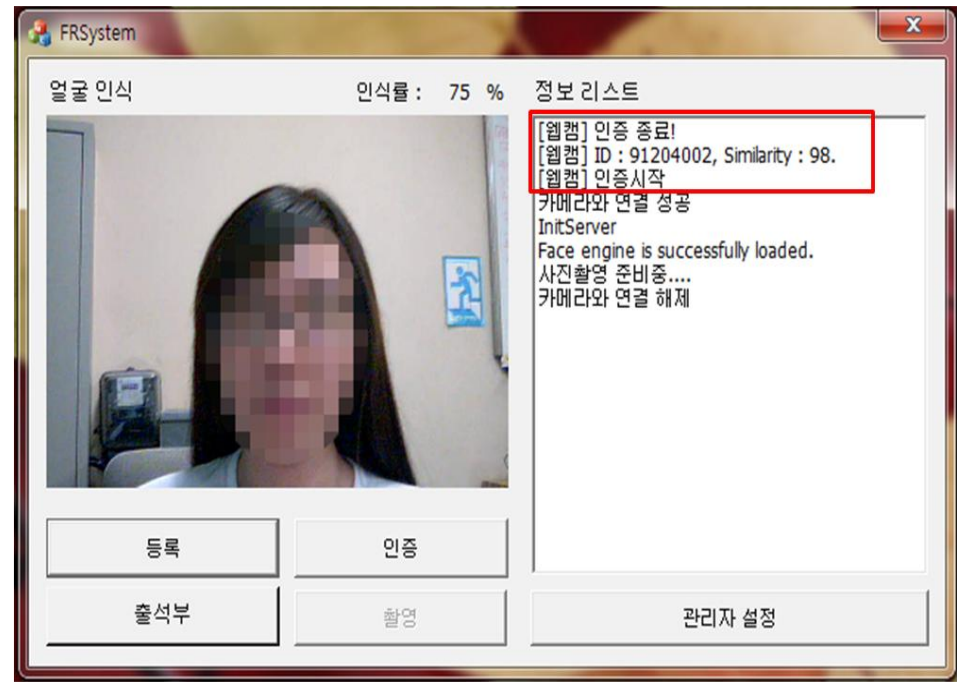

Figure 6. Face Authentication using Server 


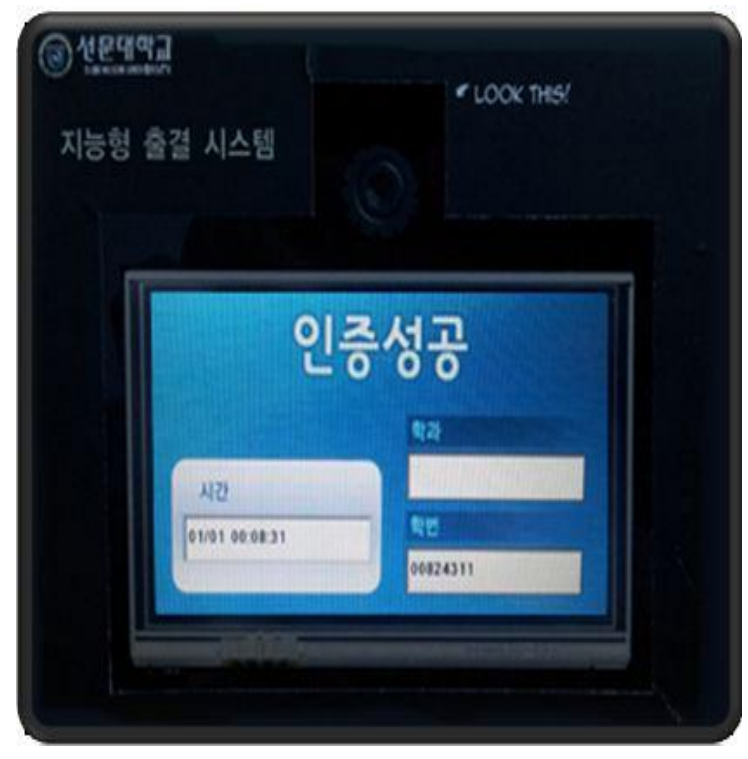

Figure 7. Face Authentication using Mobile Terminal

Figure 8 shows a student attendance book which is connected to the facial recognition system. Faces of students taken by smartphones or mobile terminals are sent to sever and go through the authentication process.

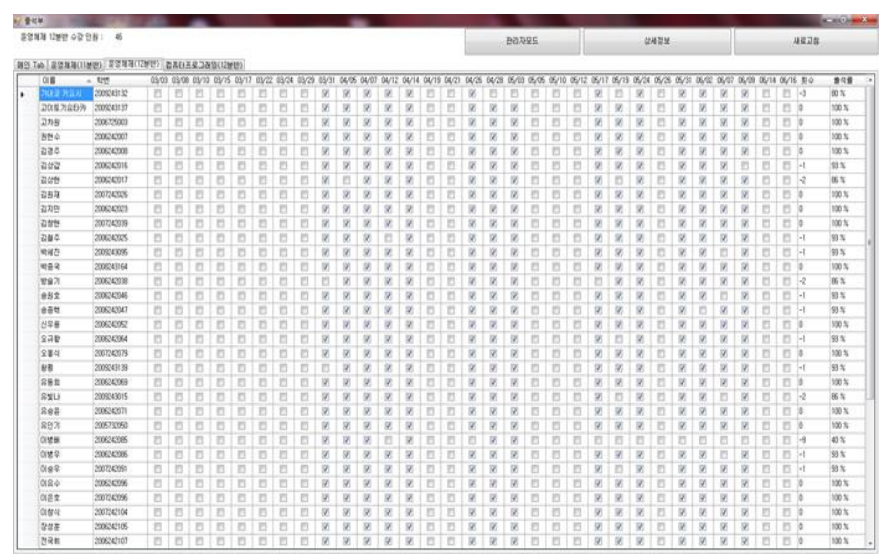

Figure 8. Student Attendance Supporting Application System

\section{Conclusions}

A face recognition system gains flexibility and cost efficiency while being integrated into a wireless network. Meanwhile, face recognition enhances the functionality and security of the wireless network. We develop a facial recognition system which uses mobile terminals and smartphones. This system sends the pictures from mobile devices to the PC server and finds the corresponding face comparing the registered data. Since we design the system for the light weight and minimize the implementation cost, we can reduce the construction cost more than $10 \%$. Our system can be applied to several areas such as indolence management, access management and attendance management. 


\section{References}

[1] http://en.wikipedia.org/wiki/Face_recognition, (2013).

[2] R. Brunelli and T. Poggio, "Fundamentals" - Computer Vision for Human-Computer Interaction, IEEE Trans. on PAMI, vol. 15, no. 10, (2003).

[3] Y. Yan and L. A. Osadciw, "Distributed wireless face recognition system", Proceedings of 2008 Multimedia Content Access: Algorithms and Systems II, (2008) January 27; San Jose, USA.

[4] M. I. Razzak, B. A. Elmogy, M. K. Khan and K. Alghathbar, "Efficient distributed face recognition in wireless sensor network", International Journal of Innovative Computing, Information and Control, vol. 8, no. 4, (2012).

[5] M. N. Gawed, O. K. R. Rahmita and F. Khalid, "Region-Based Facial Expression Recognition in Still Images", Journal of information processing systems, vol. 9, no. 1, (2013).

[6] N. Zaeri, F. Mokhtarian and A. Cherri, "Efficient face recognition for wireless surveillance systems", Proceedings of Computer Graphics and Imaging 2007, (2007) February 1, Innsbruck, Austria.

[7] Y. Yan, R. Muraleedharan and L. A. Osadciw, "Ant system based flexible and cost effective routing for wireless face recognition", Proceedings of CCNC2008, (2008) January 10, Las Vegas, USA.

[8] I. Kim, J. Shim, J. Schlessman and W. Wolf, "Remote wireless face recognition employing zigbee", Proceedings of Workshop on Distributed Smart Cameras 2006, (2006) October 1, Boulder, USA.

[9] R. E. V. Dyck and L. E. Miller, "Distributed sensor processing over an ad hoc wireless network: simulation framework and performance criteria", Proceedings of Military Communications Conference 2001, (2001) October 28, San Jose, USA.

[10] Y. Yan and L. A. Osadciw, "Fusion for Component based Face Recognition", Proceedings of CISS 07, (2007) March 2, Baltimore, USA.

[11] M. Turk and A. Pentland, "Eigenfaces for recognition”, Journal of Cognitive, vol. 3, no. 1, (1991).

\section{Authors}

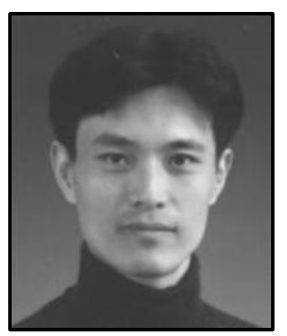

\section{KyungOh Lee}

He received his B.Sc., M.Sc., and Ph.D. in Computer Science from Seoul National University in 1989, 1994, 1999, respectively. In 1999, he joined Sun Moon University. His areas of interest are databases, realtime multimedia, and mobile computing. He is a chair of the program committee of APIS (Asian-Pacific International Symposium).

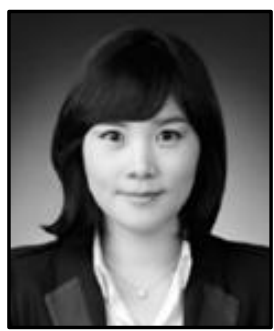

\section{YongKeum Choi}

She received her $\mathrm{RDH}, \mathrm{PhD}$ from preventive dentistry College of Dentistry, Gangnung National University 2010, 2013, respectively. In 2012, she joined Sun Moon University. Her areas of interest are public dental health, dental hygiene education, preventive dentistry, behavioral sciences for dental hygiene. 
International Journal of Bio-Science and Bio-Technology

Vol.6, No.2 (2014)

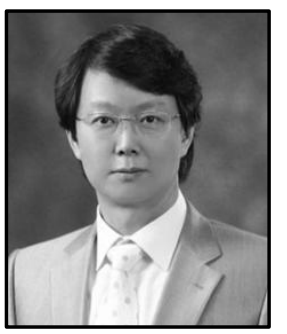

\section{YoonYoung Park}

He received his M.Sc., and Ph.D. in Computer Science from Seoul National University in 1985, 1994, respectively. In 1993, he joined Sun Moon University. His areas of interest are operating system, bioinformatics, facial recognition. 